\title{
EXAMPLES OF THE USE OF FOURIER SERIES IN STRUCTURAL OPTIMIZATION*
}

\author{
BY
}

K. BALASUBRAMANYAM AND W. R. SPILLERS

Rensselaer Polytechnic Institute

\begin{abstract}
This paper examines the use of Fourier series (finite integral transforms) in some beam-type problems of optimal structural design. In general, the series solution method reduces continuous mathematical programming problems to discrete ones. This is of particular interest when accurate results can be obtained with only a few terms of the series. Six problems of vibration and buckling are treated, most of which are not now available in the literature.
\end{abstract}

1. Introduction. There is a significant history of the application of Fourier series (more generally, integral transform) methods in applied mechanics. These methods have been most successful when applied to problems involving differential equations with constant coefficients such as beams, plates, and shells of uniform thickness. The more practical problems of variable member size have been relegated to numerical methods such as the finite-element method with a resulting wane in the more classical procedures. Notable exceptions to this are applications of the "spectral method" [1].

At first glance, integral transform methods do not deal conveniently with the products of functions which occur in problems involving differential equations with variable coefficients. Attempts to apply integral transformations in these cases invariably lead to Faltung theorems such as [2]

$$
\mathscr{F}\{F(t) \cdot G(t)\}=\int_{-\infty}^{\infty} g(\eta) f(x-\eta) d x .
$$

Here

$$
\mathscr{F}\{F(t)\}=f(\eta)
$$

indicates the operation of taking the Fourier transform $f(\eta)$ of the function $F(t)$. Similarly,

$$
\mathscr{F}\{G(t)\}=g(\eta)
$$

\footnotetext{
${ }^{*}$ Received June 20, 1985.
} 
indicates a second Fourier transform pair and

$$
f(\eta)=\frac{1}{\sqrt{2 \pi}} \int_{-\infty}^{\infty} F(t) e^{-i \eta t} d t .
$$

Sneddon [2] remarks later in his book that "Faltung theorems for finite Fourier transforms cannot be put into simple, elegant form such as hold for ordinary Fourier transforms."

The point is that when truncated Fourier series are used in practice and only a few terms are required, the products of series can be dealt with quite simply (see Appendix 1). That fact is the basis of this paper. More generally, it will be seen that Fourier series methods reduce continuous mathematical programming problems to discrete ones.

Six examples of optimization problems from the areas of vibration and buckling are discussed below. With the exception of the problem of the buckling of a pin-ended column, these designs do not now appear to be available in the literature. In all cases apparently accurate results are obtained by using two or three terms of a truncated series. This is reminiscent of some older problems of mechanics in which Fourier series methods have proved to be quite effective.

2. A vibration problem. The equation for the steady-state forced vibration of a beam can be written as

$$
\left(E I y^{\prime \prime}\right)^{\prime \prime}-\rho A \omega^{2} y=w
$$

where

$$
\begin{aligned}
& y=\text { beam (lateral) deflection, } \\
& \omega=\text { frequency of the steady-state motion, } \\
& E=\text { Young's modulus, } \\
& I=\text { beam moment of inertia } \\
& \rho=\text { beam material density } \\
& A=\text { beam area } \\
& w=\text { applied (lateral) load }
\end{aligned}
$$

In all the cases discussed in this note it will be assumed that the area and moment of inertia are related by

$$
I=\alpha A^{\nu}
$$

where $\alpha$ and $\nu$ are coefficients which depend upon the beam cross section used. In these terms it can be proposed to fix the total material volume of a simply supported beam, given the load, and look for the distribution of material $A=A(x)$ which, for example, minimizes the center displacement (maximizes the stiffness). That is, given $w, \rho, \omega, \alpha, \nu$ and $V_{0}$ (the fixed volume of material), find $A(x)$ to

$$
\text { minimize } \bar{y}=y_{x=L / 2} \text { subject to } \int_{0}^{L} A(x) d x=V_{0} .
$$

Typical of all the examples discussed in this paper, the unknown quantities are expanded in Fourier series (see Appendix 1) which are truncated, keeping two or three terms in each series. Equating coefficients of the orthogonal functions results in algebraic equations to be satisfied by these coefficients. The results are simple, discrete mathematical programming problems. 
In order to clarify the details of this method, this first example will be examined more carefully than the others. Let, for example, $I=\alpha A^{2}$. If a two-term symmetric solution is to be constructed let

$$
\begin{gathered}
y=a_{1} \sin \pi x / L+a_{3} \sin 3 \pi x / L, \\
A=\beta_{0}-\beta_{2} \cos 2 \pi x / L, \\
I=b_{0}+b_{2} \cos 2 \pi x / L+b_{4} \cos 4 \pi x / L .
\end{gathered}
$$

In order to ensure that both $I$ and $A$ are positive for all values of $0 \leqslant x \leqslant L$ when the series are truncated, it is necessary to keep an additional term in Eq. (10). The load must also be expanded in a sine series in this case. These expansions are then substituted into the equation of motion, products of sines and cosines cleared by using Eq. (A1.4), and the coefficients of the resulting sine terms equated to zero. The equation $I=\alpha A^{2}$ must also be cleared of products of sines and cosines. What results is seven unknown coefficients $a_{1}, a_{3}, \beta_{0}, \beta_{2}, b_{0}, b_{2}, b_{4}$ to be selected to satisfy six equations:

Equations of motion [Eq. (6)] -two equations,

$$
I=\alpha_{1} A^{2}
$$

Constant volume

-three equations, -one equation.

At this point the optimization problem of Eq. (7) has been reduced to a single degree of freedom problem, which can be solved, for example, by using a one-dimensional search on the coefficient $\beta_{2}$.

Table I. Simply Supported Beam-Uniform Load

\begin{tabular}{|c|c|c|c|c|}
\hline \multicolumn{3}{|c|}{ Case I } & \multicolumn{2}{c|}{$\begin{array}{c}\text { Case II } \\
I=\alpha_{1} A^{2}\end{array}$} \\
\hline no. of terms & 2 & 3 & 2 & 3 \\
\hline$A$ (in. $^{2}$ ) & $50-50 \cos \frac{2 \pi x}{l}$ & $50-33 \cos \frac{2 \pi x}{l}$ & $50-43 \cos \frac{2 \pi x}{l}$ & $50-37 \cos \frac{2 \pi x}{l}$ \\
& & $-17 \cos \frac{4 \pi x}{l}$ & & $-13 \cos \frac{4 \pi x}{l}$ \\
\hline$y_{\min }$ (in.) & 0.001527 & 0.0015512 & 0.001943 & 0.001935 \\
\hline \%reduction & 36.5 & 35.5 & 19.15 & 19.5 \\
\hline
\end{tabular}

Notes:

$* \%$ reduction $=\left(y_{\text {unif }}-y_{\min }\right) / y_{\text {unif }}$, where $y_{\text {unif }}$ is the uniform beam solution [3].

$y_{\text {unif }}=0.0024034$ in. [3]

$\mathrm{u}^{\prime}=100 \mathrm{lb} / \mathrm{ft}$

$\omega=16.66 \mathrm{rad} / \mathrm{sec}$ (half the natural frequency of the uniform beam solution)

$l=10 \mathrm{ft}$

$\rho=0.2835 \mathrm{lb} /$ in. $^{3}$ (steel)

$V_{0}=6000$ in. $^{3}$

$E=30 \times 10^{6} \mathrm{psi}$

$\alpha_{1}=1 / 6$

$\alpha_{2}=8.33$

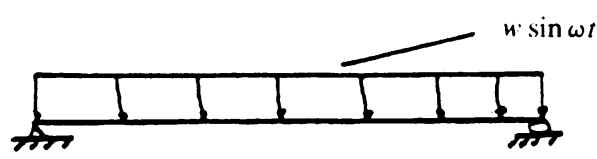

Beam Configuration 
For a "three term" solution, three terms are kept in the expansions of both $y(x)$ and $A(x)$. Eleven coefficients arise in this case, which must satisfy nine equations $\mathrm{r} \epsilon$ :ulting in two degrees of freedom and a two-dimensional search for the parameters which correspond to the minimum displacement.

Two numerical examples are indicated in Tables I and II. In the first, a simply supported beam is subjected to a harmonically varying uniform load; in the second, the same beam is subjected to a concentrated load at its center. In each case two different assumptions are made concerning the relationship between area and moment of inertia.

Table II. Simply Supported Beam-Concentrated Center Load $P$

\begin{tabular}{|c|c|c|c|c|}
\hline \multicolumn{3}{|c|}{$\begin{array}{l}\text { Case I } \\
I=\alpha_{1} A^{2}\end{array}$} & $\begin{array}{l}\text { Case II } \\
I=\alpha_{2} A\end{array}$ \\
\hline no. of terms & 2 & 3 & 2 & 3 \\
\hline$A$ (in. $^{2}$ ) & $50-50 \cos \frac{2 \pi x}{l}$ & $50-36 \cos \frac{2 \pi x}{l}-14 \cos \frac{4 \pi x}{l}$ & $50-50 \cos \frac{2 \pi x}{l}$ & $50-40 \cos \frac{2 \pi x}{l}-10 \cos \frac{4 \pi x}{l}$ \\
\hline$Y_{\text {min }}$ (in.) & 0.002148 & 0.002289 & 0.00290 & 0.002958 \\
\hline$\%$ reduction & 43.8 & 40.1 & 24.1 & 22.6 \\
\hline
\end{tabular}

Notes:

$$
\begin{aligned}
P & =1000 \\
y_{\text {unif }} & =0.00382 \mathrm{in} .
\end{aligned}
$$

See Table I for remaining parameters.

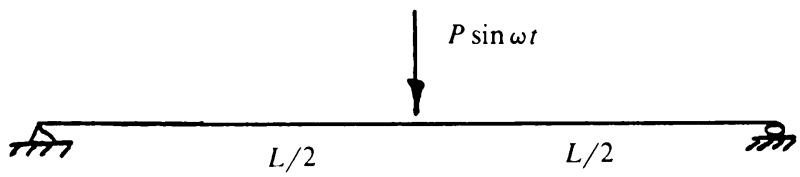

Beam Configuration

Table III. Buckling of a Pin-ended Column - Three-Term Solution

\begin{tabular}{|l|c|c|c|c|c|}
\hline & $\begin{array}{c}\text { Computed buckling } \\
\text { Load } P_{n}\end{array}$ & $\beta_{2}$ & $\beta_{4}$ & $A$ (in. $^{2}$ ) & $\mathrm{P}_{\mathrm{n}} / \mathrm{P}_{\text {unif }}$ \\
\hline $\mathrm{I}=\alpha_{2} \mathrm{~A}$ & $515.5^{k}$ & 34 & 16 & $\begin{array}{l}50-34 \cos \frac{2 \pi x}{l} \\
-16 \cos \frac{4 \pi x}{l}\end{array}$ & 1.24 \\
\hline$I=\alpha_{1} A^{2}$ & $582.5^{k}$ & 32 & 18 & $50-32 \cos \frac{2 \pi x}{l}$ & 1.40 \\
\hline
\end{tabular}

Notes:

$P_{\text {unir }}$ is the Euler buckling load $\pi^{2} E I / L^{2}$.

See Table I for a list of parameters.

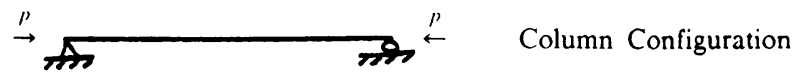


Table IV. Buckling of a pin-ended column-Comparison with available solutions

\begin{tabular}{|l|c|c|c|c|c|}
\hline \multirow{2}{*}{ Cases } & \multicolumn{2}{|c|}{ Two-term solution } & \multicolumn{2}{|c|}{ Three-term solution } & $\begin{array}{c}\text { Available } \\
\text { solution } \\
P_{F}\end{array}$ \\
\cline { 2 - 5 } & $P_{n} / P_{F}$ & $\%$ diff. & $P_{n} / P_{F}$ & $\%$ diff. & \\
\hline$I=\alpha_{2} A$ & 1.22 & -0.41 & 1.24 & 1.22 & 1.225 \\
\hline$I=\alpha_{1} A^{2}$ & 1.34 & -1.72 & 1.40 & 2.71 & 1.363 \\
\hline
\end{tabular}

Notes:

$P_{F}=$ solution given by Frauenthal [4].

$\%$ diff $=\left(P_{n}-P_{F}\right) * 100 / P_{F}$.

Table V. Simply Supported Beam-Uniform Load Mass $M$ Added at Center

\begin{tabular}{|l|c|c|c|c|}
\hline Case & \multicolumn{2}{|c|}{$I=\alpha_{1} A^{2}$} & \multicolumn{2}{c|}{$I=\alpha_{2} A$} \\
\hline no. of terms & 2 & 3 & 2 & 3 \\
\hline$A$ (in. $^{2}$ ) & $50-50 \cos \frac{2 \pi x}{l}$ & $\begin{array}{c}50-34 \cos \frac{2 \pi x}{l} \\
-16 \cos \frac{4 \pi x}{l}\end{array}$ & $50-50 \cos \frac{2 \pi x}{l}$ & $\begin{array}{c}50-37 \cos \frac{2 \pi x}{l} \\
-13 \cos \frac{4 \pi x}{l}\end{array}$ \\
\hline$y_{\min }$ (in.) & 0.0019400 & 0.00194599 & 0.00268163 & 0.0026194 \\
\hline \%reduction & 46.15 & 45.98 & 25.56 & 27.2 \\
\hline
\end{tabular}

Notes:

$r_{\text {unif }}=0.00360259$ in. [6].

See Table VI for other parameters.

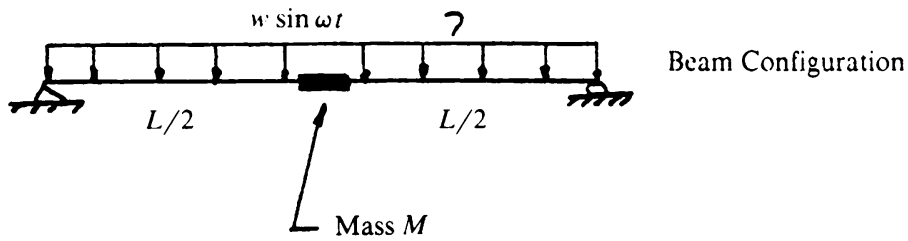

3. A buckling problem. The truncated Fourier series method described above works equally well for problems of buckling of simply supported columns. These problems can be described by the differential equation

$$
E I y^{\prime \prime}+P y=0 .
$$

Following the work of $\S 2$ above, it is possible to pose the problem of a fixed material volume for which it is desired to find the shape $A(x)$ which corresponds to the maximum buckling load. The results of this analysis are indicated in Tables III and IV, which again include two cases of different assumptions concerning the relationship between area and 
moment of inertia. In this case there is considerable literature available (e.g., [4]), which can be seen to agree closely with the approximate method used here.

4. Some more practical problems. The utility of the method described in this paper lies more with its ability to deal with problem variations that might be of practical significance than with its ability to deal with classical problems. In this vein two additional cases are treated. In the first of these a known lumped mass is added to the vibration problems of $\$ 2$ above. These results are given in Tables V and VI.

As a final example, a load is added at the midpoint of the pin-ended column of $\S 3$ above. These results are given in Table VII.

5. Concluding remarks. This paper has discussed how truncated Fourier series can be used in some structural engineering applications of optimal design. In the examples cited, accurate results seem to require only two or three terms in each series, but this of course reflects the fact that these two or three terms mirror the optimal shape closely. In retrospect, experience with structures would lead the engineer to believe that this would be so.

Table VI. Simply Supported Beam-Concentrated Center Load Mass $M$ Added at Center

\begin{tabular}{|c|c|c|c|c|}
\hline Case & \multicolumn{2}{|c|}{$I=\alpha_{1} A^{2}$} & \multicolumn{2}{c|}{$I=\alpha_{2} A$} \\
\hline no. of terms & 2 & 3 & 2 & 3 \\
\hline$A$ (in. $^{2}$ ) & $50-50 \cos \frac{2 \pi x}{l}$ & $\begin{array}{c}50-36 \cos \frac{2 \pi x}{l} \\
-14 \cos \frac{4 \pi x}{l}\end{array}$ & $50-50 \cos \frac{2 \pi x}{l}$ & $\begin{array}{c}50-39 \cos \frac{2 \pi x}{l} \\
-11 \cos \frac{4 \pi x}{l}\end{array}$ \\
\hline$y_{\min }$ (in.) & 0.0027250 & 0.0028741 & 0.0039773 & 0.0040039 \\
\hline \%reduction & 52.41 & 49.81 & 30.54 & 30.07 \\
\hline
\end{tabular}

Notes:

\%reduction $=\left(y_{\text {unif }}-y_{\min }\right) * 100 / v_{\text {unif }}$.

$y_{\text {unif }}=0.0057257$ in. [6].

$\omega=9.59 \mathrm{rad} / \mathrm{sec}$ (half the natural frequency of the uniform beam solution [6]).

$M=$ added center mass $634.7 \mathrm{lb}-\mathrm{sec}^{2} /$ in.

See Table 1 for other parameters.

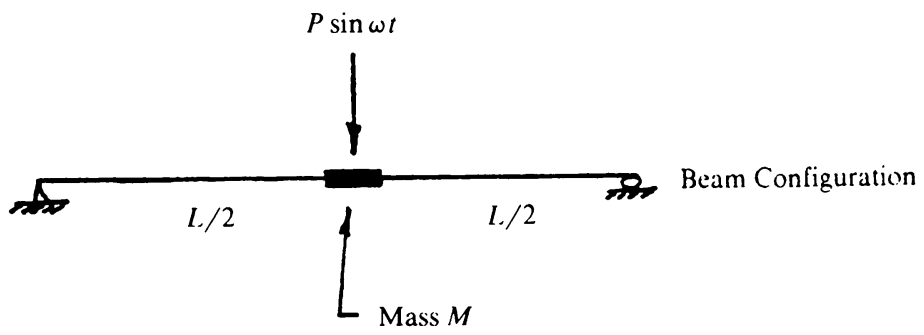


More complex structures with other boundary conditions will be more difficult to deal with. But series solutions have certain advantages over purely numerical methods such as ease in dealing with lumped masses, providing analytical expressions for shape, and in some cases use trivial search procedures. At the very least they offer an additional tool to the engineer.

Appendix 1: Products of Fourier series. There are some cases in which the products of Fourier series can be reduced directly to a single series. For example, if a beam deflection $y(x)$ and its moment of inertia $I(x)$ are written in series form as

$$
\begin{aligned}
& y=\sum_{n=1}^{\infty} a_{n} \sin n \pi x / L, \\
& I=\sum_{m=0}^{\infty} b_{m} \cos m \pi x / L,
\end{aligned}
$$

the product $I y$ can be reduced to a single series

$$
\begin{aligned}
I y & =\left(\sum_{n=1}^{\infty} a_{n} \sin n \pi x / L\right)\left(\sum_{m=0}^{\infty} b_{m} \cos m \pi x / L\right) \\
& =\sum_{n=1}^{\infty} c_{n} \sin n \pi x / L,
\end{aligned}
$$

with the coefficients $c_{i}$ functions of $a_{i}$ and $b_{i}$. To do so only requires use of the addition formula

$$
2 \cdot \cos x \cdot \sin y=\sin (x+y)-\sin (x-y) \text {. }
$$

Column configuration

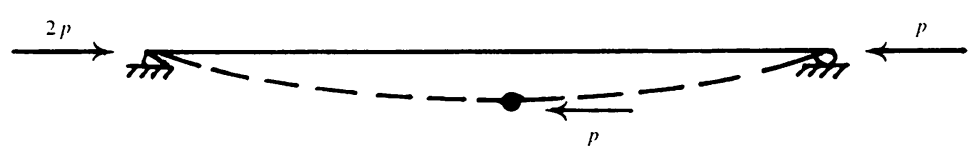

Case: $I=\alpha_{1} A^{2}$, two-term solution

Optimum Design:

Buckling load: $7141^{\wedge}$

Shape: $A=50-50 \cos 2 \pi x / l$

\%increase over uniform column: $26 \%$

Uniform column:

Buckling load [5]: $5660^{k}$

Approximate solution*: $5712^{h}$

\footnotetext{
* This approximate solution is obtained by assuming constant area in the two-term Fourier series solution.
} 
The expression for the coefficients $c_{i}$ follows as

$$
\begin{aligned}
I y= & \frac{1}{2} \sum_{n=1}^{\infty} \sum_{m=0}^{\infty} a_{n} b_{m}[\sin (n+m) \pi x / L-\sin (m-n) \pi x / L] \\
= & \sum_{k=1}^{\infty} \sin k \pi x / L \frac{1}{2} \sum_{l=-k}^{k-2} \frac{a_{k-l}}{2} \frac{b_{k+l}}{2} \\
& +\sum_{l=1}^{\infty} \sin l \pi x / L\left(-\frac{1}{2}\right) \sum_{k=l+2}^{\infty} * \frac{a_{k-l}}{2} \frac{b_{k+l}}{2} \\
& +\sum_{l^{\prime}=1}^{\infty} \sin l^{\prime} \pi x / L \sum_{k=l^{\prime}}^{\infty} \frac{a_{k+l^{\prime}}}{2} \frac{b_{k-l^{\prime}}}{2},
\end{aligned}
$$

where

$$
k=m+n, \quad l=m-n, \quad l^{\prime}=-l,
$$

and $\Sigma^{*}$ indicates a sum whose terms are incremented by two. The expressions for each $c_{i}$ follow directly from Eq. (A1.5) by collecting the last sum in each of the above terms. They are awkward and will not be written out here. In applications in which only a few series terms are involved, they can be obtained most easily from Eq. (A1.4) directly without recourse to the general expression for $c_{i}$.

Other applications follow routinely. For example, if the beam area $A(x)$ is expanded in a cosine series and if it is assumed that $I$ and $A$ are related as $I=\alpha A^{2}$, the relationship between $I$ and $A$ can be resolved by using the addition formula

$$
2 \cdot \cos x \cdot \cos y=\cos (x+y)+\cos (x-y) \text {. }
$$

\section{REFERENCES}

[1] Hans Schamel and Klaus Elsasser, The Application of the spectral method to nonlinear wave propagation, J. Comput. Phys. 22, pp. $501-516$ (1976)

[2] Ian M. Sneddon, Fourier transforms, McGraw-Hill, New York (1951)

[3] S. Timoshenko, Vibration problems in engineering, Van Nostrand, New York (1937)

[4] James C. Frauenthal, Constrained optimal design of columns against buckling, J. Structural Mech. 1, no. 1, 79-89 (1972)

[5] S. Timoshenko, Strength of materials, Part 2, Advanced theory and problems, Robert E. Krieger. Huntington, New York (1976)

[6] Robert D. Blevins, Formulas for natural frequencies and mode shape, Van Nostrand Reinhold, New York (1979) 